\title{
Year in Review: Synopsis of Selected Articles in Neuroanesthesia and Neurocritical Care from 2018
}

\author{
${ }^{1}$ Department of Anesthesia \& Pain Medicine, University of Toronto, \\ Toronto Western Hospital, Toronto, Ontario, Canada \\ 2Department of Anesthesiology, Perioperative and Pain Medicine, \\ University of Manitoba, Management, Health Sciences Centre, \\ Manitoba, Canada
}

Kyle J. Rogan ${ }^{1}$ Tumul Chowdhury ${ }^{2}$ Lakshmikumar Venkatraghavan ${ }^{1}$

\begin{abstract}
Address for correspondence Lakshmikumar Venkatraghavan, MBBS, MD, DNB, FRCA, FRCPC, 2McL-405, Toronto Western Hospital, 399, Bathurst Street, Toronto, Ontario, Canada M5T 258 (e-mail: Lashmi.Venkatraghavan@uhn.ca).
\end{abstract}

J Neuroanaesthesiol Crit Care 2019;6:18-23

\section{Dexmedetomidine and Pain in Neurosurgery}

Dexmedetomidine (DEX) is a highly selective alpha-2 agonist that is commonly used in neuroanesthesia due its ability to provide sedation without respiratory depression. In addition, it acts as a mild analgesic and neuroprotective agent, and decreases the anesthetic requirements. The opioid sparing effect of DEX has been investigated in various small randomized controlled trials (RCTs); this meta-analysis presents a trial sequential analysis of 11 RCTs and updates a previous meta-analysis published in 2014. A total of 674 patients undergoing intracranial procedures were identified. DEX use was associated with less intraoperative fentanyl requirement (mean difference [MD]: -127.75), lower visual analog scale score (MD: -1.54 ), less nausea (odds ratio [OR]: 0.59), and less shivering (OR: 0.31). Postoperative opioid use was also decreased slightly (standard $\mathrm{MD}=-0.88$ ); more patients would be needed to consider the result reliable. Regarding perioperative hemodynamics, the incidence of hypertension (OR: 0.32) and tachycardia (OR: 0.31) was higher in the control group than the DEX group. However, there were no significant differences in hypotension and bradycardia between the groups. Interestingly, the extubation time was noted to be 5.46 minutes shorter in the group receiving DEX. ${ }^{1}$

Overall, this meta-analysis shows that DEX can be utilized as an opioid sparing analgesic in neurosurgical

\section{received}

December 4, 2018 accepted after revision

January 3, 2019

published online

March 5, 2019 cases. Opioid-free to minimal opioid regimens offer less opioid-related side-effects and may provide better neurological examination conditions with fewer negative cognitive effects. It is important to mention that this study did not reach its required sample size, and heterogeneity of data could not be eliminated completely. Moreover, the study period was limited to the postanesthesia care unit. The role of scalp blocks in the included trials was also not highlighted. More work is required to determine the effect of DEX on reducing opioid-induced hyperalgesia and chronic pain in neurosurgical patients. A multicenter RCT would help delineate the ideal procedures, optimal dosing, role of pre-emptive analgesic administration, and side-effects of DEX.

\section{Restrictive Transfusion Protocol in Traumatic Brain Injury}

Restrictive transfusion strategies have become commonplace; however, there has been a reluctance to adopt the practice in neurosurgical patients. The authors compared patients admitted to their institution with traumatic brain injury (TBI) from before and after implementing a change in the hemoglobin transfusion threshold from $<10 \mathrm{~g} / \mathrm{dL}$ to $<7 \mathrm{~g} / \mathrm{dL}$ in their hospital-wide TBI protocol. The protocol was otherwise static over this time period. Patients aged $<16$ years, who died $<24$ hours after admission, and who were admitted to a
Copyright $\odot 2019$ Indian Society of Neuroanaesthesiology and Critical Care
License terms

10.1055/s-0039-1679135

ISSN 2348-0548.

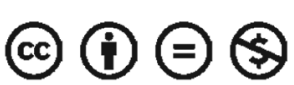


3-month transition between hemoglobin threshold targets were excluded. Data on 1,565 patients were obtained from a prospectively collected database. There was no difference in the incidences of acute respiratory distress syndrome and venous thromboembolism, or length of stay in intensive care and days of mechanical ventilation between the groups. The lower threshold group experienced statistically fewer days of fever. Subgroup analysis on patients presenting with a Glasgow Coma Scale (GCS) score $\leq 8$ produced similar results although the benefit with respect to fewer fever days was lost. There was a $25 \%$ decrease in the proportion of patients who received a transfusion. Cost analysis suggested a transfusion-related savings of $\$ 242$ to 394 (2016 USD) per patient; for patients with GCS score $\leq 8$, $\$ 1049$ to 1710 in savings was seen. The study supports the safety of implementing restrictive transfusion practices in a TBI population after acute resuscitation and stabilization is achieved. ${ }^{2}$

\section{Tranexamic Acid in Intracerebral Hemorrhage}

Tranexamic acid (TA) has been shown to reduce intracranial bleeding and further hematoma expansion after traumatic intracranial hemorrhage (ICH). The feasibility of an RCT looking at TA in spontaneous ICH was confirmed in a single-center study (TICH-1) in 2014. Sprigg et al conducted an international (124 hospitals in 12 countries) randomized, double-blind, placebo-controlled trial comparing early treatment with TA with standard supportive care in patients with spontaneous ICH after an ischemic stroke. A total of 2,325 patients were randomized to receive a $1 \mathrm{~g}$ intravenous TA bolus followed by an additional $1 \mathrm{~g}$ infusion over 8 hours or placebo within 8 hours of symptom onset. The groups were balanced and adherence to the prescribed protocol was $95 \%$. Notably, the primary outcome, functional outcome at 90 days as assessed by the modified Rankin Score, was not statistically different between the groups. Although the TA group showed a significant reduction in early deaths (adjusted OR: 0.73), the effect did not persist for 90 days. It is also important to mention that fewer patients in the TA group had hematoma expansion and the volume of expansion was comparatively smaller at 24 hours. The incidence of serious adverse events at 90 days was also significantly lower in TA group (TA 45 vs. placebo $48 \%, p=0.0393$ ). Thirty-six percent of patients were recruited within 3 hours and there was no heterogeneity in treatment effect when $<3$ hours versus $>3$ hours to treatment was compared. Venous thromboembolism, arterial occlusion, and seizures were not increased by TA. Overall, this large trial demonstrated a lack of efficacy of TA in ICH; however, the broad inclusion criteria increased heterogeneity, and inclusion of patients with severe or nonsurvivable ICH might have diluted the observed effect. In addition, the effect of TA on functional outcomes of different types and locations of spontaneous ICH has yet to be explored. As the use of TA does not evoke major adverse events, further investigation into its potential benefit in this and other intracranial bleeding is merited. ${ }^{3}$

\section{General Anesthesia versus Sedation in Endovascular Thrombectomy}

Simonsen et al conducted the third (after AnStroke and SIESTA) RCT aimed at resolving the uncertainty regarding the impact of general anesthesia (GA) on outcomes in endovascular thrombectomy (ET) for stroke. In this single-center study, 128 patients undergoing ET for acute ( $<6$ hours) ischemic stroke were randomized to have the procedure under GA or under sedation. Patients in the GA group were intubated using alfentanil, propofol, and suxamethonium and then maintained on propofol $(2-10 \mathrm{mg} / \mathrm{kg} / \mathrm{hr})$ and remifentanil $(0.2-1 \mu \mathrm{g} / \mathrm{kg} / \mathrm{min})$. The non-GA group was sedated using fentanyl boluses and a propofol infusion (1-2 $\mathrm{mg} / \mathrm{kg} / \mathrm{hr}$ ). Additional targets included a systolic blood pressure $>140 \mathrm{~mm} \mathrm{Hg}$, mean arterial pressure $>70 \mathrm{~mm} \mathrm{Hg}$, and extubation after the procedure in the neurointerventional suite. The end-point evaluation was blinded, and outcomes were analyzed using intention to treat principles. Despite reperfusion being more successful in the GA group, there was no difference between groups for the primary outcome, infarct size on magnetic resonance imaging conducted 48 to 72 hours postprocedure (median [interquartile range] growth 8.2 vs. $19.4 \mathrm{~mL}, p=0.10$ ). There was a shift toward improved modified Rankin Scores at 90 days (OR: 1.91) with $\mathrm{GA}$. Time to reperfusion was similar between both treatment arms (212 vs. 216 minutes). Although a mean arterial pressure fall > 20\% was more frequent in the GA group $(87.7 \%$ vs. $34.9 \%$ ), the duration of time it was below $70 \mathrm{~mm} \mathrm{Hg}$ was not statistically different. This study supports the use of GA as an alternative to conscious sedation assuming that delays in treatment are minimized and hemodynamic parameters are controlled. Future large RCTs should explore why functional outcomes and infarct size vary depending on type of anesthesia used in various previous trials. What is the missing link? Is it really a general anesthetic induced stress, case delays, alterations in cerebral blood flow related to hypotension or hypocapnia, or simply biased selection contributing to the worse outcomes reported in a few trials? ${ }^{4}$

\section{Outcomes for Endovascular Thrombectomy in Stroke Under General Anesthesia}

Whether or not GA imposes adverse outcomes after ET in stroke is still a matter of debate. To explore these concerns, seven trials (1,764 patients) were included in this meta-analysis. They assessed functional outcome in ischemic stroke patients with large vessel anterior circulation occlusion undergoing ET under GA, versus thrombectomy not under GA (with or without sedation) versus standard care (i.e., no thrombectomy), stratified by the use of GA versus standard care. Several investigators created the international Highly Effective Reperfusion Evaluated in Multiple Endovascular Stroke (HERMES) Collaboration, and pooled their respective patient-level data for the analysis. A robust statistical assessment using multivariate regression and propensity-score stratification was used. Outcomes after GA were significantly worse (corrected OR: 1.53 ) compared 
with the non-GA group; 18 of every 100 patients undergoing ET under GA showed a worse functional outcome. It was further estimated that 10 out of these 18 patients would not be functionally independent. There was no difference in vessel rupture or the rate of symptomatic ICH among all three groups (non-GA, GA, and standard medical care). Importantly, GA did not completely negate the benefit of ET over standard medical therapy (corrected OR: 1.52). The meta-analysis was limited in that the included studies were randomized on the basis of type of stroke treatment received (ET with standard medical care in anterior circulation stroke) and not on the type of anesthesia used for the procedure. The approach to anesthetic care was not randomized and therefore, many patients could have received GA because it was medically indicated, which could have impacted the outcome. In addition, patients in the non-GA group may have had minimal to significant sedation with variable drugs and hemodynamic targets. Given the results of this study, it would appear prudent to avoid GA when reasonably possible in this patient population. ${ }^{5}$

\section{Predictors of Outcome after General Anesthesia for Endovascular Thrombectomy}

Athiraman et al hoped to identify anesthetic factors associated with improved outcome after ET for stroke performed under GA to form the basis for recommendations on management. Adult patients who underwent ET for stroke between December 2010 and June 2014 were included. Over the specified time period almost all procedures were performed under GA. Eighty-eight patients met the selection criteria, $22 \%$ of which had a modified Rankin Score $(\mathrm{mRS}) \leq 2$ at the time of discharge and $15 \%$ of which died in hospital. Factors those were not predictive of outcome included age, sex, stroke distribution, comorbid illnesses, hemorrhage, pneumonia, vasopressor use, type of induction or maintenance agents, and anesthetic duration. Predictors of poor outcome included higher presenting National Institute of Health Stroke Scale (NIHSS) scores and preprocedural intubation in the emergency department. Chronic beta-blocker therapy was associated with good outcome ( $m R S \leq 267 \%$ vs. mRS > $233 \%$ ). No blood pressure threshold was independently associated with good outcome. Of note, $88 \%$ of patients had a systolic blood pressure during the procedure below the currently recommended target of 140 $\mathrm{mm} \mathrm{Hg}$. Higher mean end-tidal carbon dioxide $(49 \pm 8 \mathrm{~mm}$ Hg) (OR: 2.24) and extubation after endovascular treatment (OR: 26.31) were the only independent predictors of good outcome after adjusting for age and NIHSS scores. These results direct further attention toward ventilation strategies and timely extubation; they need to be confirmed with larger prospective studies. ${ }^{6}$

\section{Hypertonic Saline versus Mannitol for Supratentorial Tumors}

Ali et al compared 3\% hypertonic saline (HS) with 20\% mannitol (M) for the reduction in intracranial pressure (ICP) in patients with supratentorial tumors in this single-center, prospective, randomized, double-blinded study. ${ }^{7}$ In contrast to other previous trials, this study included the placement of an intraparenchymal ICP monitor and strictly controlled physiologic variables including anesthetic depth using entropy. Only patients with significant mass effect (GordonFiring score >1) were considered. ${ }^{8}$ Parenchymal probes were placed adjacent to the tumor by a neurosurgeon. After assessment of baseline physiologic variables and ICP, either $5 \mathrm{~mL} / \mathrm{kg}$ of $3 \% \mathrm{HS}$ or $20 \% \mathrm{M}$ was administered over 15 minutes. ICP values were obtained every 5 minutes during the infusion and for the following 30 minutes. After exclusions, only 20 patients in the $\mathrm{M}$ arm and 19 patients in the HS arm completed the study. Patients in the HS arm had a comparatively higher central venous pressure $(10.1 \mathrm{mmHg}$ vs. $9.6 \mathrm{~mm} \mathrm{Hg}$ ), lower pulse pressure variation (6.4\% vs. 7.2\%) at 45 minutes after infusion $(p<0.05)$, compared with the $\mathrm{M}$ arm. HS produced a greater decrease in ICP from baseline $(-38.4 \%$ vs. $-30.3 \%, p<0.05)$. The median difference in the ICP decrease between the two groups was only $1 \mathrm{~mm} \mathrm{Hg}$ in favor of HS, but when accounting for the starting ICPs, HS was $8 \%$ more effective than $\mathrm{M}$. Comparisons made in patients with more grossly elevated ICP may result in a greater observed difference. ICP values were not correlated well with brain relaxation scores, and brain relaxation scores were comparable between the two groups. It is also noteworthy to mention that five patients in $\mathrm{M}$ group and only one patient in HS group required a second dose. This is a small single-center study and a much larger, high-quality RCT is required to provide further clinically relevant information regarding comparative efficacy and the associated safety profile of $\mathrm{M}$ and HS in this population.

\section{Dreaming and Awareness with Dexmedetomidine and Propofol}

Prior to this study, there was uncertainty as to whether or not dreaming reported after anesthesia occurs only during recovery after termination of the anesthetic or during the anesthetic itself. The authors investigated the presence of awareness (connected consciousness) and dreaming (disconnected consciousness) during and after recovery from target-controlled infusions of DEX and propofol. Forty-seven healthy young adult male volunteers were randomized in permuted blocks to receive either drug. Loss of responsiveness was determined by assessing the ability of participants to respond to semantically congruent or incongruent prerecorded sentences after stepwise achievement of predetermined plasma concentrations of the study drugs. Semi-structured interviews using a modified Brice questionnaire were conducted when the participant could be aroused. Complete loss of consciousness was then achieved by increasing drug concentrations by $50 \%$. A final interview was conducted after recovery. An emotional sound stimulus (such as a baby crying) was played prior to each return of responsiveness and recovery. Two independent judges analyzed and sorted interview reports into several categories including whether or not the recalled memories 
happened during a period of responsiveness and if they reflected dreaming, awareness, or memory incorporation. Participants who received DEX were much more successfully awakened and reported experiences from the unresponsive period more often than those who received propofol. Dreaming was common in both groups (93\% with DEX and $72 \%$ with propofol) and occurred in the recovery period as well as during anesthetic infusion. Awareness of the research environment was noted in $22.6 \%$ of those receiving DEX and $4 \%$ of those receiving propofol, but in each case these reports were associated with brief arousals from unresponsiveness. None of the participants directly referenced the emotional sound even when prompted by interview. They were, however, able to recognize familiar stimuli ( $42 \%$ with DEX vs. $15 \%$ with propofol), implying that explicit memory and conscious connectedness was present during the infusions. ${ }^{9}$

\section{Propofol Pharmacodynamics during Awake Craniotomy}

Soehle et al looked to identify the bispectral index (BIS) values and plasma concentrations ( $C_{\text {plasma }}$ ) of propofol during return of consciousness and the point at which mapping was possible for 13 patients undergoing awake craniotomy. ${ }^{10}$ They employed an asleep-awake-asleep anesthetic technique method: a propofol infusion was initially titrated to BIS of 40 to 60 for scalp infiltration, Mayfield fixation, craniotomy, and dural opening. The infusion was then discontinued and the time to response to verbal command was determined. Propofol $C_{\text {plasma }}$ measured using high-pressure liquid chromatography was compared with the concentrations predicted by the Marsh and Schnider models. BIS at return of consciousness was $77 \pm 7$ with $C_{\text {plasma }}$ of $1.2 \pm 0.4 \mu \mathrm{g} / \mathrm{mL}$. Brain mapping occurred as soon as patients were alert enough to proceed; BIS at the initiation of mapping was $92 \pm 6$ with $C_{\text {plasma }}$ of $0.8 \pm 0.3 \mu \mathrm{g} / \mathrm{mL}$. Return of consciousness from a $C_{\text {plasma }}$ of $2.5 \pm 0.8 \mu \mathrm{g} / \mathrm{mL}$ took $10.5 \pm 3.2$ minutes while testing was possible $23 \pm 12$ minutes after discontinuation of the infusion. The Marsh model significantly overestimated the $C_{\text {plasma }}$ at both return of consciousness $(1.9 \pm 0.4 \mu \mathrm{g} / \mathrm{mL})$ and mapping $(1.3 \pm 0.5 \mu \mathrm{g} / \mathrm{mL})$, while the Schnider model predictions were similar to the measured values $(1.4 \pm 0.4 \mu \mathrm{g} / \mathrm{mL}$ at return of consciousness and $1.0 \pm 0.4 \mu \mathrm{g} / \mathrm{mL}$ at mapping). The Marsh model significantly overestimated propofol concentrations at critical periods during awake craniotomy. The Schnider models showed a higher accuracy and lower bias suggesting that it should be preferentially used in this setting.

\section{Venous Air Embolism and Head-Up Position}

Venous air embolism (VAE) is a devastating complication during craniotomy in head-up position. The authors of this study sought to define the relationship between the degree of head up positioning and VAE. Fifty ASA I and II patients presenting for resection of infratentorial lesions were prospectively enrolled into one of the two groups-30-degree head-up (group 1), and 45-degree-s head-up (group 2) semi-sitting positions. Assignment was based on the position felt to obtain the optimal surgical exposure given the location of a patient's lesion. Intraoperative assessment for VAE was conducted via transesophageal echocardiogram (TEE) and a grading system from 0 (none) to 4 (life threatening) was employed to classify the severity based on the amount of bubbles seen and the presence of end-tidal carbon dioxide or hemodynamic disturbances. The 30-degree head-up position was associated with significantly lower rates $(22 \%$ vs. $62.5 \%$, OR: 0.16 ) and lower severity (grade 2 or higher in $8 \%$ vs. 50\%, OR: 0.08) of VAE. VAE was also more common during resection of meningiomas. Only two patients developed VAE-related hypotension requiring hemodynamic support; both were from group 2. There were no life-threatening events in either group. The authors advocate for the continued use of the semi-sitting position in select situations given its advantages for surgical exposure and the ability to mitigate morbidity and mortality through rapid diagnosis and treatment with current monitoring techniques. ${ }^{11}$

\section{Evoked Potentials during Carotid Endarterectomy}

There is no universally agreed method for identifying cerebral hypoperfusion during carotid cross-clamping for endarterectomy. At the authors' institution, neurologic assessment was facilitated by an asleep-awake-asleep technique referred to as cooperative patients under GA (CPGA) until 2009 when they transitioned to using somatosensory-evoked potentials (SSEPs) and motor-evoked potentials (MEPs). Marinò et al conducted a retrospective review of their practice to identify the incidence of technical failures for both approaches to neuromonitoring and the shunt rate, complications, and surgical duration. Three hundred thirty-one patients who underwent carotid endarterectomy from 2008 to 2011 were identified, 232 using evoked potential monitoring, and 102 using CPGA. For CPGA, patients were intubated and the anesthetic maintained with propofol and remifentanil. During the awake portion, the propofol infusion was interrupted and remifentanil titrated to patient distress. Neurologic testing was completed with the endotracheal tube in-situ every minute after clamping for 5 minutes and then every 3 minutes until 15 minutes after clamp removal. Development of a neurologic deficit despite blood pressure optimization prompted shunting. With evoked potential monitoring, shunting occurred if there was a reproducible decrement of $>50 \%$ in the contralateral SSEP amplitudes or MEP amplitudes $<25 \%$ of baseline. Neurologic monitoring via evoked potentials had a significantly lower rate of technical failure $(0.4 \%$ vs. 3.9\%, OR: 0.11) compared with CPGA. Fifteen out of hundred patients in the CPGA group exhibited neurologic dysfunction with clamping, while 25/231 had significant changes to their evoked potentials. Only one patient in the study had persistent postoperative cerebral ischemia; in this case, the changes in evoked potentials failed to resolve despite shunt placement. Substantially lower operative and cross clamp times were seen after introduction of evoked potential monitoring. Overall, the results support the use of evoked potentials as a means of monitoring for cerebral hypoperfusion in patients undergoing carotid endarterectomy. ${ }^{12}$ 


\section{Deep Brain Stimulation for Vegetative State}

Recovery from a persistent vegetative state (VS) is rare. Some promising reports showing improvement in VS with deep brain stimulation (DBS) have been published; however, these previous studies focused mainly on TBI patients. Ngwenya et al looked to further investigate the potential benefits of DBS in this population as well as in patients with VS and minimally conscious state (MCS) secondary to hypoxic encephalopathy. Forty-nine patients were screened for inclusion. To qualify, they needed to meet criteria for VS/MCS; demonstrate recordable SSEPs, MEPs, and brain stem auditory evoked potentials; have periods of electroencephalogram desynchronization over 12 to 24 hours of continuous monitoring; and have intact cerebral glucose metabolism as assessed via positron emission tomography. Fourteen patients underwent stereotactic electrode placement targeting the centromedianparafascicular complex of the thalamic intralaminar nucleus of the left or less injured hemisphere. Stimulation was performed for 30 minutes every 2 hours during the day starting on postoperative day 3. The Rappaport Disability Rating Scale (range: $0-30$ with high values reflecting more severe disability) and the Coma/Near-Coma Scale were used to evaluate patients preoperatively, weekly for 3 months postinsertion, and then monthly until study completion (38-60 months). Three of the four patients with MCS returned to full awareness, two of which were able to live mostly independent. For these two patients, awareness was achieved 30 to 45 days and a "walk state" was achieved 88 to 210 days after initiating treatment. One of the 10 patients with VS responded with return to full awareness although he is still bedridden. Return to a full level of awareness took 295 days. Anoxic myoclonic jerks also disappeared after initiation of DBS. Although the authors are unable to rule out the role of spontaneous recovery in their observations, the rate of recovery observed appeared substantially faster than typically seen. As those with recovery had the shortest time from injury to DBS treatment, initiation of therapy when the injury is remote may confer no benefit. ${ }^{13}$

\section{Narrative Reviews of Interest}

Several excellent review articles, focusing on topics of particular interest to the neuroanesthesiologists, were published last year. Kisilevsky et al conducted a comprehensive review of transfusion thresholds in neurosurgical patients and recommended an optimal hemoglobin concentration of 9.0 to $10.0 \mathrm{~g} \mathrm{dL}^{14}$ Smith $^{15}$ reviewed neuromonitoring in TBI and Harvey et al have provided some guidance to physicians caring for those with devastating brain injury via a consensus statement including direction on the decision to transition to palliative care. ${ }^{16}$ Cordonnier et al have summarized the current management of acute $\mathrm{ICH} .{ }^{17}$ Finally, excellent discussions of the anesthetic care for patients undergoing novel functional and laser ablation neurosurgical procedures were provided by Dunn et al and Jimenez-Ruiz et al. ${ }^{18,19}$

\section{Funding \\ None.}

\section{Conflict of Interest}

None declared.

\section{References}

1 Liu Y, Liang F, Liu X, Shao X, Jiang N, Gan X. Dexmedetomidine reduces perioperative opioid consumption and postoperative pain intensity in neurosurgery: a meta-analysis. J Neurosurg Anesthesiol 2018;30(2):146-155

2 Ngwenya LB, Suen CG, Tarapore PE, Manley GT, Huang MC. Safety and cost efficiency of a restrictive transfusion protocol in patients with traumatic brain injury. J Neurosurg 2018;128(5):1530-1537

3 Sprigg N, Flaherty K, Appleton JP, et al; TICH-2 Investigators. Tranexamic acid for hyperacute primary IntraCerebral Haemorrhage (TICH-2): an international randomised, placebo-controlled, phase 3 superiority trial. Lancet 2018;391(10135):2107-2115

4 Simonsen CZ, Yoo AJ, Sørensen LH, et al. Effect of general anesthesia and conscious sedation during endovascular therapy on infarct growth and clinical outcomes in acute ischemic stroke: a randomized clinical trial. JAMA Neurol 2018;75(4):470-477

5 Campbell BCV, van Zwam WH, Goyal M, et al; HERMES collaborators. Effect of general anaesthesia on functional outcome in patients with anterior circulation ischaemic stroke having endovascular thrombectomy versus standard care: a meta-analysis of individual patient data. Lancet Neurol 2018;17(1):47-53

6 Athiraman U, Sultan-Qurraie A, Nair B, et al. Endovascular treatment of acute ischemic stroke under general anesthesia: predictors of good outcome. J Neurosurg Anesthesiol 2018;30(3):223-230

7 Ali A, Tetik A, Sabanci PA, et al. Comparison of 3\% hypertonic saline and $20 \%$ mannitol for reducing intracranial pressure in patients undergoing supratentorial brain tumor surgery: a randomized, double-blind clinical trial. J Neurosurg Anesthesiol 2018;30(2):171-178

8 Hernández-Palazón J, Fuentes-García D, Doménech-Asensi P, Piqueras-Pérez C, Falcón-Araña L, Burguillos-López S. A comparison of equivolume, equiosmolar solutions of hypertonic saline and mannitol for brain relaxation during elective supratentorial craniotomy. $\mathrm{Br} \mathrm{J}$ Neurosurg 2016;30(1):70-75

9 Radek L, Kallionpää RE, Karvonen M, et al. Dreaming and awareness during dexmedetomidine- and propofol-induced unresponsiveness. Br J Anaesth 2018;121(1):260-269

10 Soehle M, Wolf CF, Priston MJ, et al. Propofol pharmacodynamics and bispectral index during key moments of awake craniotomy. J Neurosurg Anesthesiol 2018;30(1):32-38

11 Türe H, Harput MV, Bekiroğlu N, Keskin Ö, Köner Ö, Türe U. Effect of the degree of head elevation on the incidence and severity of venous air embolism in cranial neurosurgical procedures with patients in the semisitting position. J Neurosurg 2018;128(5):1560-1569

12 Marinò V, Aloj F, Vargas M, et al. Intraoperative neurological monitoring with evoked potentials during carotid endarterectomy versus cooperative patients under general anesthesia technique: a retrospective study. J Neurosurg Anesthesiol 2018;30(3):258-264

13 Chudy D, Deletis V, Almahariq F, Marčinković P, Škrlin J, Paradžik V. Deep brain stimulation for the early treatment of the minimally conscious state and vegetative state: experience in 14 patients. J Neurosurg 2018;128(4):1189-1198 
14 Kisilevsky A, Gelb AW, Bustillo M, Flexman AM. Anaemia and red blood cell transfusion in intracranial neurosurgery: a comprehensive review. Br J Anaesth 2018;120(5):988-998

15 Smith M. Multimodality neuromonitoring in adult traumatic brain injury: a narrative review. Anesthesiology 2018;128(2):401-415

16 Harvey D, Butler J, Groves J, et al. Management of perceived devastating brain injury after hospital admission: a consensus statement from stakeholder professional organizations. Br J Anaesth 2018;120(1):138-145
17 Cordonnier C, Demchuk A, Ziai W, Anderson CS. Intracerebral haemorrhage: current approaches to acute management. Lancet 2018;392(10154):1257-1268

18 Dunn LK, Durieux ME, Elias WJ, Nemergut EC, Naik BI. Innovations in functional neurosurgery and anesthetic implications. J Neurosurg Anesthesiol 2018;30(1):18-25

19 Jimenez-Ruiz F, Arnold B, Tatsui CE, Cata JP. Perioperative and anesthetic considerations for neurosurgical laser interstitial thermal therapy ablations. J Neurosurg Anesthesiol 2018;30(1):10-17 